\title{
10 Systems approach to quantify the global omega-3 fatty acid 11 cycle
}

12 Helen A. Hamilton ${ }^{*}, 1$, Richard Newton ${ }^{2}$, Neil A. Auchterlonie ${ }^{3}$, Daniel B. Müller ${ }^{1}$

$13{ }^{1}$ Norwegian University of Science and Technology (NTNU), Industrial Ecology Programme,

14 Sem Sælands Vei 7, Trondheim, Norway

15 Institute of Aquaculture, University of Stirling, Stirling, FK9 4LA, UK.

$16{ }^{3}$ IFFO, Marine Ingredients Organisation, Printworks, Unit C, 22 Amelia Street, London,

17 SE17 3BZ

18 Key words: Aquaculture, Fisheries, EPA, DHA, Material flow analysis

19 Abstract

20 Long-chain omega-3 fatty acids, eicospeantaenoic and docosahexaenoic acids, are essential

21 components of human diets and some aqua/animal feeds - but are sourced from finite marine

22 fisheries, in short supply and deficient in large parts of the world. We use quantitative

23 systems analysis to model the current global EPA/DHA cycle and identify options for

24 increasing supply. Opportunities lie in increased by-product utilization and food waste

25 prevention. Economic, resource, cultural and technical challenges need, however, to be

26 overcome.

27 Main Text

28 Long-chain omega-3 fatty acids (FA), in particular eicosapentaenoic (EPA) and 29 docosahexaenoic (DHA) acid, are essential components of human diets due to their role in 30 visual and neurological development in infants and the vast range of cognitive, 31 cardiovascular and psychological benefits for adults. ${ }^{1}$ The daily recommended intake of 32 EPA/DHA ranges between 250 and $1000 \mathrm{mg}$ for healthy adults, with higher DHA 33 requirements for pregnant and lactating women. ${ }^{1}$ The primary dietary source for EPA/DHA is 34 fish; however, fish themselves are inefficient at producing EPA/DHA and instead accumulate 35 them through the food chain from primary producers. ${ }^{2}$ 
36 First estimates show that aquaculture, fisheries and other marine sources supply 0.8 million

37 tonnes of EPA/DHA per year for human consumption. ${ }^{2}$ This is below the human nutritional

38 demand of 1.4 million tonnes required to supply the global population with $500 \mathrm{mg}$

39 EPA+DHA daily and will be further exacerbated by population growth. EPA/DHA

40 deficiencies have been observed worldwide and particularly affect populations located in

41 North America, central Europe, the Middle East, India, Brazil and the U.K., with regional and

42 socio-economic differences seen within the countries. ${ }^{3}$ Filling the EPA/DHA supply gap is

43 unlikely to occur through capture fisheries, due to $63 \%$ of fish stocks being considered

44 exploited and in need of rebuilding. ${ }^{4}$ Aquaculture can increase the supply of EPA/DHA,

45 however, many farmed species require the input of fish meal (FM) and fish oil (FO) sourced

46 from capture fisheries and seafood byproducts to meet their nutritional needs and maintain

47 the FA profile of the fish. ${ }^{5}$ Due to the scarcity and increasing price of marine oils, the

48 aquafeed industry has reduced FM and FO inclusion by partial substitution with plant

49 ingredients. $^{6}$ Thus, aquaculture production has grown at $5.8 \%$ per annum, without

50 considerably increasing FM and FO consumption. ${ }^{7}$ However, reduced FM and FO inclusion

51 has affected the FA profile of certain fed species (e.g. salmonids), with lowered EPA/DHA

52 contents. $^{6}$

53 The growing EPA/DHA supply gap, related potential human health consequences and the

54 need to protect marine ecosystems makes it essential to optimize the management of long-

55 chain omega-3 FA, considering all relevant intervention options and evaluating their

56 combined effects. Here, we use a systems approach and quantify the global EPA and DHA

57 cycle to i) provide a comprehensive problem description to improve overall resource

58 efficiency and ii) identify system-wide opportunities and challenges for meeting the human

59 EPA/DHA demand. We, thereafter, aim to inform decision makers on the current EPA/DHA

60 status, its drivers and the most effective intervention options at a global level. 


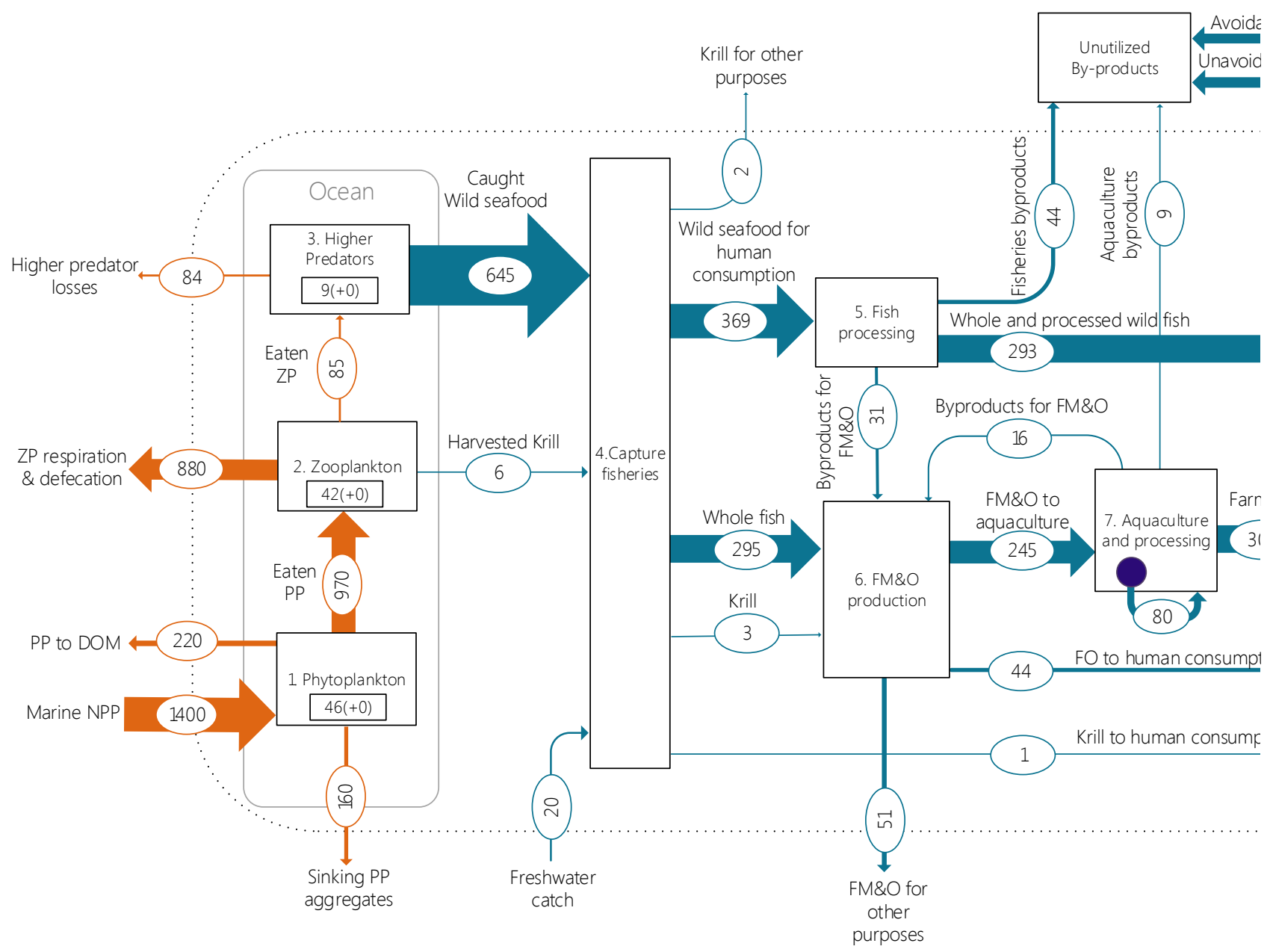

63 Figure 1. Global EPA and DHA balance; Orange arrows in Mt, Blue arrows in kt EPA+DHA/yr;

64 Purple dot denotes net endogenous EPA/DHA production by fish; NPP = Net primary production; PP 65 = Phytoplankton; DOM = Dissolved organic matter; $\mathrm{ZP}=$ zooplankton; FM\&O = fish meal and oil. 66 Mass balance inconsistencies due to i) rounding errors and ii) uncertainty All flows in process 6 were 67 independently calculated and the remaining mass balance inconsistency is less than $1 \%$ of total flows 68 in this process. Net endogenous production in the ocean system is not visualized. 
69 We find that between net primary production (NPP) and higher predators, approximately

$7090 \%$ of EPA/DHA is lost via respiration, defecation and deaths, indicating large trophic

71 losses up the food chain (figure 1). The zooplankton and phytoplankton stocks are of

72 comparable sizes (approximately 40 Mt EPA+DHA), with no net yearly addition to stock.

73 Caught wild seafood accounts for $0.04 \%$ of the EPA/DHA produced via NPP. Approximately

74 half of harvested marine EPA/DHA is managed through FM and FO production (primarily

75 for aquaculture consumption, figure 2 top) and half for direct human consumption.

76 Despite aquaculture being a major consumer of EPA/DHA, it is also a major producer via 77 non-fed species, such as molluscs and carp, accumulating EPA/DHA from their environment 78 and/or endogenous production through the elongation of shorter-chained FA. Freshwater fish 79 are better at elongation compared to marine fish due to unique enzymes and desaturase genes 80 that allow for EPA/DHA synthesis. ${ }^{8}$ In contrast, fed high-trophic salmonid species i) 81 consume a high proportion of aquaculture's use of FM and FO (58\% and 22\%, respectively, 82 in 2015), ii) have EPA/DHA retention rates varying from 30 to $75 \%$ and iii) are inefficient at 83 FA elongation ${ }^{9}$, but also supply EPA/DHA through a farmed product based on an otherwise 84 under-utilized wild fish resource.

85 We find the supply of EPA/DHA for human consumption is $420 \mathrm{kt} / \mathrm{year}$ or $149 \mathrm{mg}$ 86 EPA+DHA/capita daily, representing 30\% of global demand. We, therefore, confirm the 87 supply gap identified by Tocher et al. ${ }^{2}$ but find it to be twice as large as previous estimates. 88 Significant losses occur due to unavoidable and avoidable food waste (114 and $105 \mathrm{kt}$ $89 \mathrm{EPA}+\mathrm{DHA} / \mathrm{yr}$, respectively) and unutilized fish processing byproducts (53 kt EPA+DHA/yr), 90 with the largest losses in Asia (figure 2, bottom). 
Fish oil consumption by sector

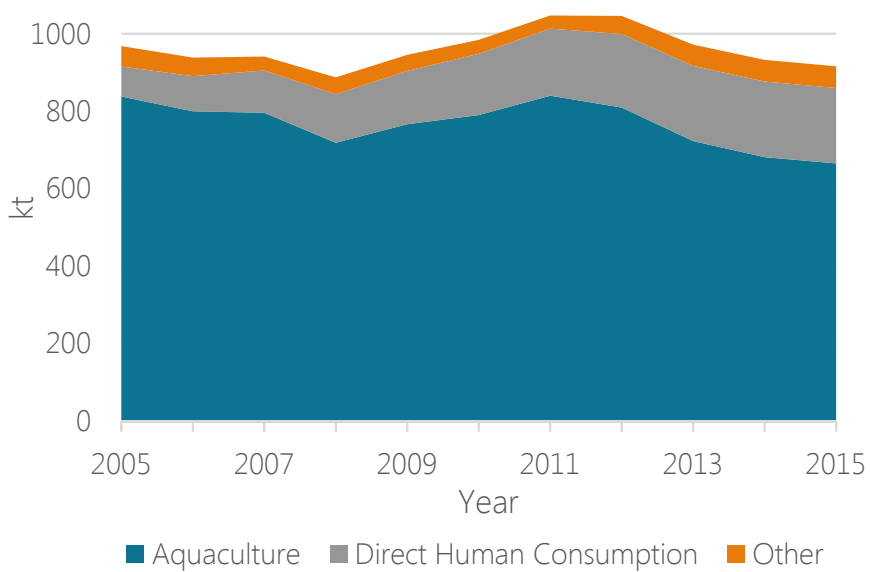

Global fishmeal consumption by sector

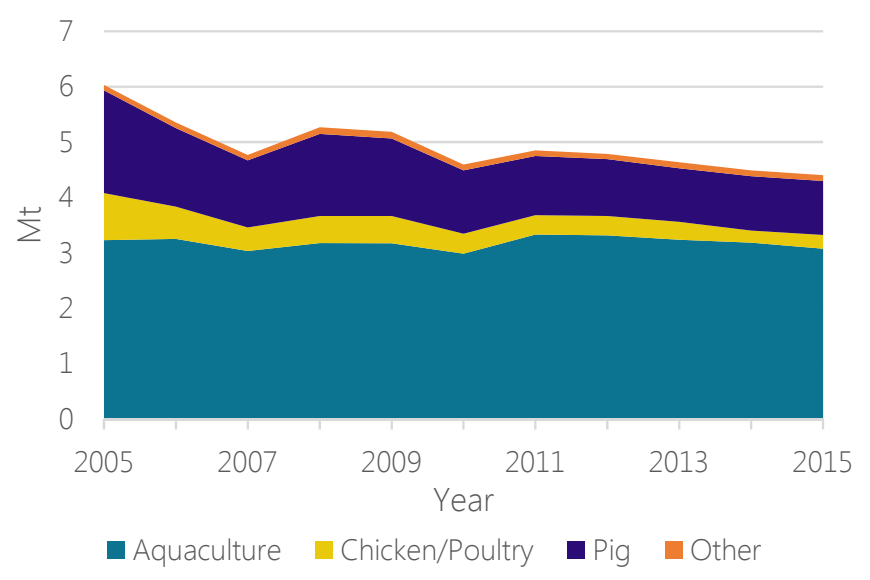

100
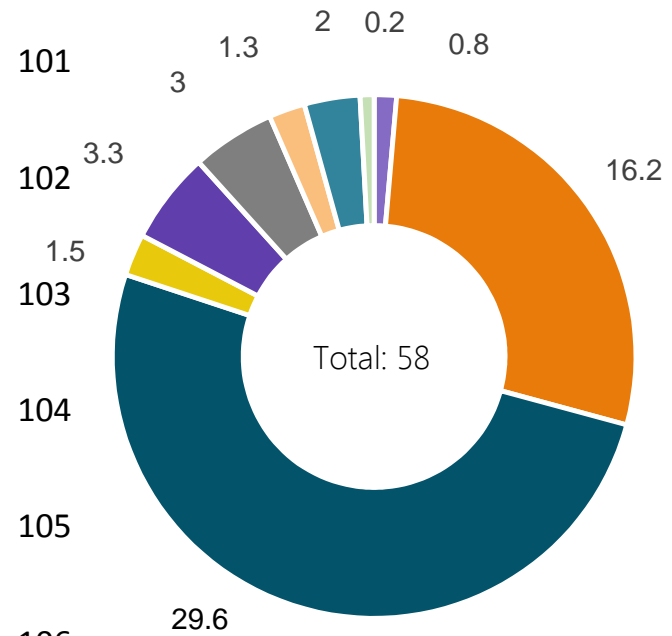

106

107

108

109

110

111 While many options exist to fill the EPA/DHA gap, each has challenges. Aquaculture's

112 strategic FM and FO in feed use at key life-stages can i) influence the EPA/DHA utilization

113 efficiency by farmed fish and ii) optimize the benefits of marine ingredients from a fish and

114 human health perspective, e.g. finishing diets to increase EPA/DHA towards harvest time. ${ }^{10}$

115 Fish stock recovery could increase long-term fish yields and the EPA/DHA supply (albeit 
116 with likely short-term decreases). ${ }^{4}$ However, forage fish harvesting may have a lowered

117 effect on stock size as compared to environmental factors that affect reproductive success. ${ }^{11}$

118 With the krill harvesting rate ( 300,000 tonnes biomass in 2018) being below the catch limit

119 of 5.6 million tonnes annually as defined by Commission for the Conservation of Antarctic

120 Marine Living Resources, increasing krill catch for use as feed could substantially increase

121 the EPA/DHA supply. ${ }^{12}$ However, Antarctic krill harvesting operations face challenges

122 related to geography and costs, and effective stock management is imperative to ensure

123 sustainable harvesting levels.

124 Avoiding trophic losses could increase supply by i) consuming EPA/DHA from a lower

125 trophic level (e.g. seaweeds, krill and bivalve mollusks), ii) increasing non-fed fish farming

126 and/or iii) diverting more wild catch to human consumption through direct consumption or

127 oil supplementation produced from these species. However, for this to prove effective, the

128 digestibility, bioavailability and efficacy of EPA/DHA in these products need to be

129 understood (e.g. the bioavailability of FA in fish oil is lower than fish ${ }^{13}$ ) and although the

130 nutraceutical market is strong, the wild fish market depends on factors including, amongst

131 others, the catch quality, acceptance and temporal challenges, i.e. seasonal surplus of fish

132 catch that cannot be absorbed by the market. ${ }^{14}$ In addition, logistical challenges exist for the

133 distribution to populations that are EPA/DHA deficient. ${ }^{15}$

134 Improved by-product utilization and food waste avoidance can substantially increase the 135 supply of EPA/DHA while reducing waste. Processing by-products can be used for FM and 136 FO production for aquafeed and/or human consumption provided the regulatory frameworks 137 are followed. ${ }^{16}$ However, a major challenge is collection and processing, as by-products are 138 often geographically dispersed. For example, Asia, where most of the by-product potential is 139 concentrated (figure 2 bottom), has the culture of buying fish whole and disposing of by140 products at the household level. ${ }^{17}$ Centralized fish processing is needed to recover by- 
141 products in this region but would require a substantial cultural shift in the way fish is

142 consumed. Food waste prevention is also an effective means for increasing supply, as it 143 avoids the unnecessary use of EPA/DHA to produce food that is wasted.

144 Future options to produce EPA/DHA include large-scale production of natural and 145 genetically modified (GM) microalgae, microbacteria and higher plants. However, current 146 technologies and concerns about GM material limit volume of supply, their cost-effectiveness 147 and widespread penetration into the market, ${ }^{19}$ although regulatory challenges related to GM 148 feed use are primarily constrained to Europe. ${ }^{20}$

\section{Sources}

$150 \quad$ 1. $\quad$ Kris-Etherton, P.M., Grieger, J.A. \& Etherton, T.D. Prostaglandins Leukot. Essent.

$151 \quad$ Fat. Acids 81, 99-104 (2009).

152 2. Tocher, D.R. Aquaculture 449, 94-107 (2015).

153 3. Stark, K.D., Van Elswyk, M.E., Higgins, M.R., Weatherford, C.A. \& Salem, N. Prog. $154 \quad$ Lipid Res. 63, 132-152 (2016).

155 4. Worm, B. et al. Science (80-. ). 325, 578-585 (2009).

156 5. Tocher, D.R. Aquac. Res. 41, 717-732 (2010).

157 6. Sprague, M., Dick, J.R. \& Tocher, D.R. Sci. Rep. 6, 1-9 (2016).

158 7. FAO (Rome, Italy, 2016).at <http://www.fao.org/fishery/sofia/en>

159 8. Rodrigues, B.L. et al. PLoS One 12, 1-15 (2017).

1609 9. Tocher, D.R. Rev. Fish. Sci. 11, 107-184 (2003).

161 10. Codabaccus, M.B., Ng, W.K., Nichols, P.D. \& Carter, C.G. Food Chem. 141, 236-244 162 (2013).

163 11. Hilborn, R. et al. Fish. Res. 191, 211-221 (2017).

164 12. CCAMLR Comm. Conserv. Antarct. Mar. Living Resour. 29 (2018).

165 13. Schram, L.B. et al. Food Res. Int. 40, 1062-1068 (2007).

166 14. Alder, J. \& Pauly, D. (Vancouver, Canada, 2006).at $<$ https://open.library.ubc.ca/cIRcle/collections/facultyresearchandpublications/52383/it ems/1.0074759>

171

15. Stark, K.D., Van Elswyk, M.E., Higgins, M.R., Weatherford, C.A. \& Salem, N. Prog. Lipid Res. 63, 132-152 (2016).

16. EC Council Off. J. EU 147, 1-40 (2001). 
172 17. Wang, F., Zhang, J., Mu, W., Fu, Z. \& Zhang, X. Food Control 20, 918-922 (2009).

18. Hamilton, H.A., Peverill, M.S., Müller, D.B. \& Brattebø, H. Environ. Sci. Technol. 49, (2015).

19. Qi, B.X. et al. Nat. Biotechnol. 22, 739-745 (2004).

20. Spicer, A. \& Molnar, A. Biology (Basel). 7, 21 (2018).

\section{Acknowledgements}

We would like to thank Erik Olav Gracey (Biomar) and Yngvar Olsen (NTNU) for their useful aquaculture insights and fruitful discussions. This research was supported by the research project MIRA (Microbially produced raw materials for aquafeed. MIRA is funded by the Research Council of Norway.

\section{Author contributions}

H.H. and D.M. designed the study. H.H., R.N. and N.A. quantified the system and conducted the analysis. H.H. made the figures. H.H., R.N., N.A. and D.M. contributed to the data interpretation. H.H. wrote the paper. H.H., R.N., N.A. and D.M. contributed to manuscript editing.

\section{Competing financial interests}

The authors declare no competing interests.

\section{Correspondence}

Correspondence should be addressed to Helen Hamilton; helen.a.hamilton@ntnu.no

\section{Methods}

We used a multi-layer material flow analysis framework (ML-MFA) to quantify the stocks and flows of EPA/DHA throughout our defined system. The 'mother' layer contains the biomass system (tonnes wet weight/yr) and the 'child' layer includes the sum of EPA and DHA balance (tonnes EPA+DHA/yr). From a mass balance standpoint, quantifying the EPA/DHA content of biological organisms is a methodological challenge due to i) marine and freshwater species storing EPA/DHA within their lipids and, thus, metabolizing them as 
199 an energy source and ii) organisms endogenously producing EPA/DHA through the 200 elongation of alpha-linolenic acid (ALA, 18:3n-3) at various rates depending on, amongst 201 others, the species, time of the year and habitat. ${ }^{21}$ Therefore, unlike substances (i.e. chemical 202 elements), EPA/DHA can be created or destroyed, which limits mass balance conservation 203 when modeling and makes it necessary to consider production and destruction. Preliminary 204 estimates have shown endogenous EPA/DHA production to contribute little to the EPA/DHA 205 supply from farmed fish (i.e. EPA/DHA consumed by aquaculture equals the EPA/DHA 206 contents of the produced fish). ${ }^{22}$ However, for certain species, endogenous EPA/DHA 207 production can be potentially significant, especially for bivalve mollusks and carp. ${ }^{23}$ 208 Therefore, we accounted for this by calculating the net EPA/DHA production of each 209 biological process for which EPA/DHA can be created/destroyed. We assumed processes that 210 mechanically transform the flows (i.e. fish processing) do not affect the EPA/DHA content of 211 the biomass.

212 We defined the system to include the natural and anthropogenic stocks and flows of 213 EPA/DHA. Freshwater ecosystem food chains were not considered due to their minor role 214 relative to the marine ecosystem and limited data availability; however, we included the 215 EPA/DHA contained in freshwater fish capture and freshwater aquaculture. In addition, we 216 did not consider natural export from marine to terrestrial ecosystems, e.g. due to the 217 consumption of drifted algae by lizards, birds and other terrestrial animals, as preliminary 218 estimates (24 kt EPA+DHA/yr) have shown this to be insignificant relative to the overall 219 marine food web. ${ }^{24}$

220 Primary data are sourced from scientific publications, reports, statistics and industry data 221 from the International Marine Ingredients Organization (IFFO). Ocean carbon flows are 222 based on Stock et al. ${ }^{25}$ and represent a 20 year average (1994-2014). The long time frame 223 minimizes the uncertainty related to yearly variations in primary production due to, e.g., El 
224 Nino events ${ }^{26}$. Capture data is based primarily on the FAO dataset FishStat and includes an 225 average between 2009 and 2013 to normalize yearly variations. Due to the large number of 226 species, we only accounted for the top 20 fish, cephalopod and crustacean species caught and 227 farmed in each geographical region. EPA/DHA calculations are performed at a species level. 228 However, we accounted for all wild and farmed bivalve mollusks and plants. Overall, we 229 accounted for over 90\% of fishery and aquaculture production. Avoidable food waste is 230 defined to include all edible food that was wasted at the household level. Unavoidable food 231 waste includes the remaining inedible fraction, such as peels, shells and bones. Further 232 information regarding the methods can be found in the supplementary information.

\section{Data Availability}

234 This work uses data collected from a variety of sources, both proprietary and freely available. 235 See references in the supplementary information for data specification. All figures are based 236 on this collected dataset and geographically aggregated data will be made available upon 237 request from the corresponding author.

238

239 1. Veloza, A.J., Chu, F.L.E. \& Tang, K.W. Mar. Biol. 148, 779-788 (2006).

240 2. FAO GLOBEFISH - Anal. Inf. world fish trade (2017).at <http://www.fao.org/in241 action/globefish/fishery-information/resource-detail/en/c/338773/>

242 3. FAO GLOBEFISH - Anal. Inf. world fish trade (2017).

243 4. Gladyshev, M.I., Arts, M.T. \& Sushchik, N.N. Lipids Aquat. Ecosyst. 179-210 (2009).

244 5. Stock, C.A., Dunne, J.P. \& John, J.G. Prog. Oceanogr. 120, 1-28 (2014).

245 6. Strutton, P.G. \& Chavez, F.P. J. Geophys. Res. 105, 260987-26101 (2000). 


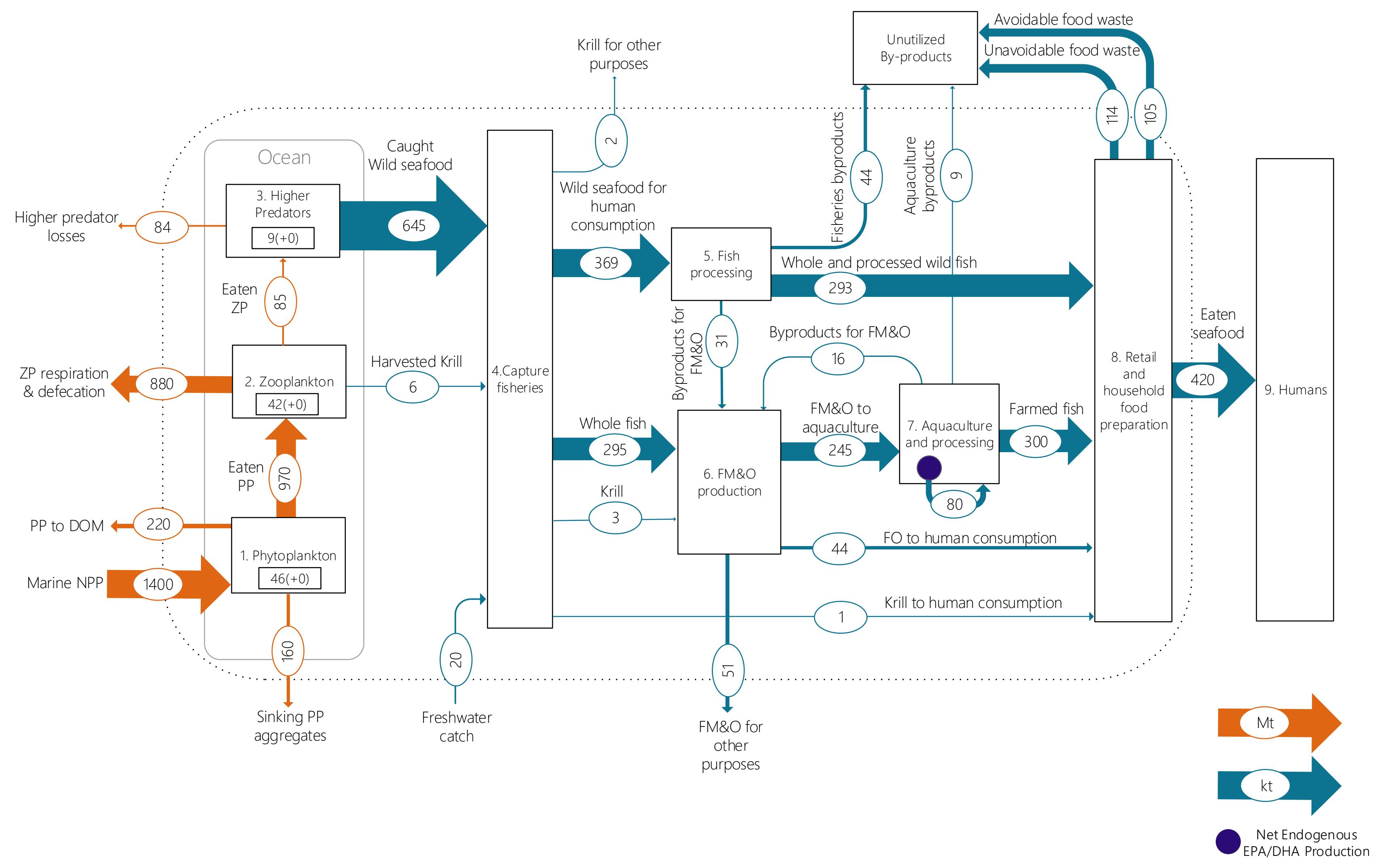


Fish oil consumption by sector

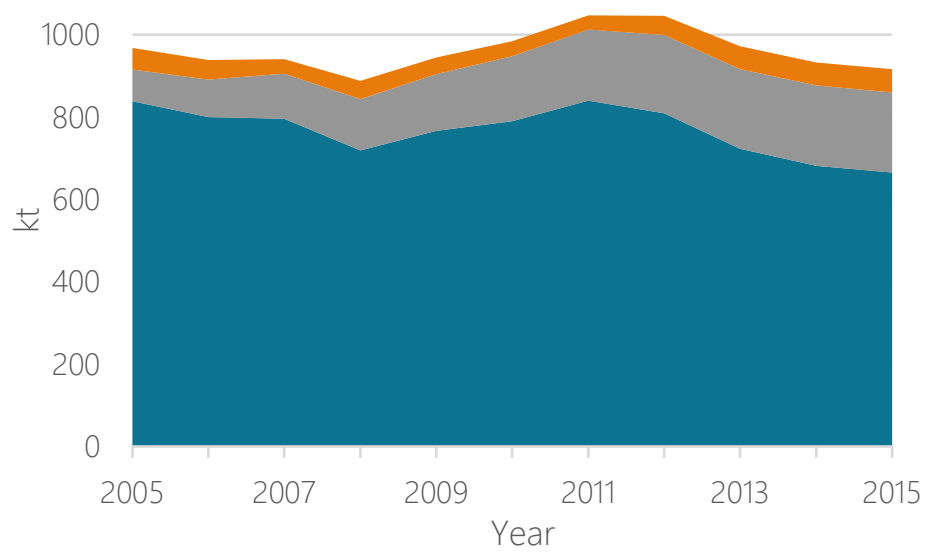

- Aquaculture Direct Human Consumption ather
Global fishmeal consumption by sector

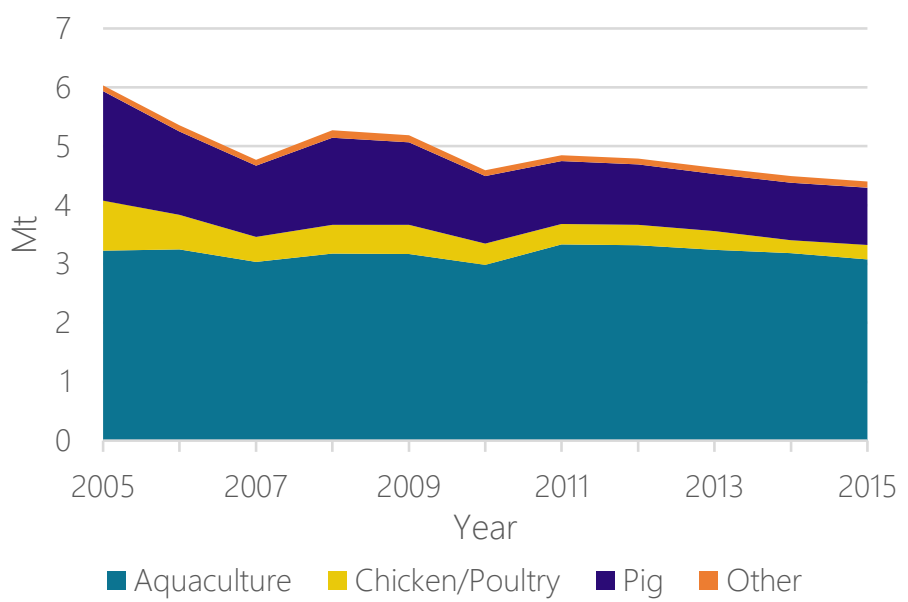

Fisheries

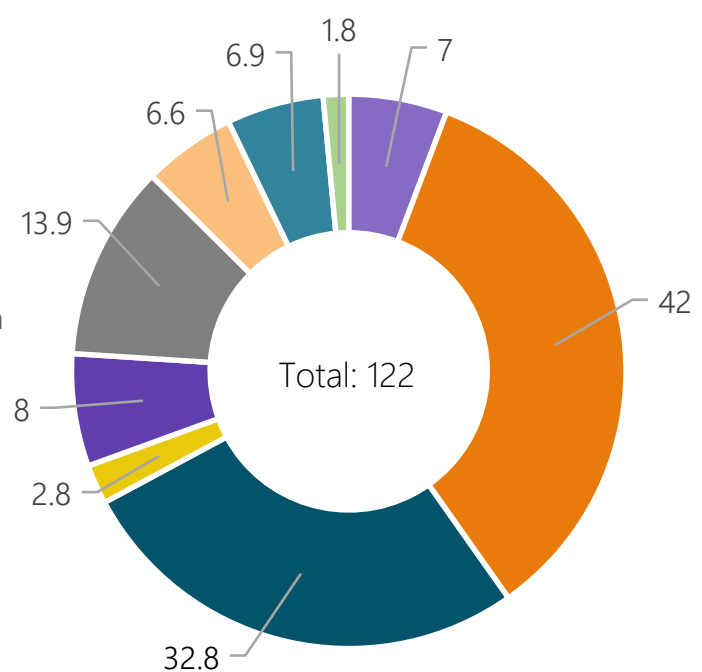




\section{A. Flat Files}

\begin{tabular}{|l|l|l|l|}
\hline Item & Present? & $\begin{array}{l}\text { Filename } \\
\text { This should be the name } \\
\text { the file is saved as when it } \\
\text { is uploaded to our system, } \\
\text { and should include the file } \\
\text { extension. The extension } \\
\text { must be .pdf }\end{array}$ & $\begin{array}{l}\text { A brief, numerical description of file contents. } \\
\text { i.e.: Supplementary Figures 1-4, Supplementary Discussion, and } \\
\text { Supplementary Tables 1-4. }\end{array}$ \\
\hline Supplementary Information & Yes & $\begin{array}{l}\text { Hamilton_Supplements. } \\
\text { pdf }\end{array}$ & $\begin{array}{l}\text { Supplementary Figure 1, Supplementary Methods, } \\
\text { Supplementary Discussion, and Supplementary Tables 1-3. }\end{array}$ \\
\hline Reporting Summary & Yes & Reporting_summary.pdf & \multicolumn{2}{|l}{}
\end{tabular}

\section{Source Data}

Complete the Inventory below for all Source Data files.

\begin{tabular}{|l|l|l|}
\hline $\begin{array}{l}\text { Parent Figure or } \\
\text { Table }\end{array}$ & $\begin{array}{l}\text { Filename } \\
\text { This should be the name the file is saved as } \\
\text { when it is uploaded to our system, and } \\
\text { should include the file extension. i.e.: } \\
\text { Smith_SourceData_Fig1.xls, or Smith_ } \\
\text { Unmodified_Gels_Fig1.pdf }\end{array}$ & $\begin{array}{l}\text { Data description } \\
\text { e.g.: Unprocessed Western Blots and/or gels, Statistical Source } \\
\text { Data, etc. }\end{array}$ \\
\hline Source Data Fig. 1 & Hamilton_SourceData_Fig1.xlsx & Numerical data used to generate figure 1 \\
\hline Source Data Fig. 2 & Hamilton_SourceData_Fig2.xlsx & Numerical data used to generate figure 2, top and bottom \\
\hline
\end{tabular}

